\title{
Cortisol, testosterone, and pain levels among patients undergoing McKenzie therapy and suboccipital relaxation
}

\author{
Corresponding author: \\ Paweł Kiczmer \\ Department of Medical \\ and Molecular Biology, \\ Medical University of Silesia \\ Zabrze, Poland \\ e-mail: \\ pawel.kiczmer@protonmail.com
}

Medical Research Journal 2020;

Volume 5, Number 1, 34-40

10.5603/MRJ.a2020.0007

Copyright (C) 2020 Via Medica

ISSN 2451-2591

\begin{abstract}
Sedentary lifestyle and the development of consumer electronics, often associated with a faulty posture, are widespread factors contributing to cervical spine dysfunction (CSD). The purpose of our study is to compare two methods of physical therapy of CSD: suboccipital relaxation and the McKenzie method. Their effect on perceived pain level and life quality was assessed using VAS and NDI scores. Serum levels of biochemical stress indicators like testosterone and cortisol were also evaluated. Eighty-six adult patients were divided into two groups: Group A and Group B. Group A included 42 patients treated using the McKenzie method. Group B consisted of 44 patients who underwent suboccipital relaxation. The therapy in both groups included three treatment sessions over a six-week period. Testosterone and cortisol levels were assessed using the ELISA technique. Pain evaluation was performed using the Visual Analogue Scale (VAS). The disability level was evaluated with the Neck Disability Index (NDI). In both groups, a similar improvement in VAS and NDI scores was observed. A distinct cortisol level decrease in patients subjected to the suboccipital relaxation was noticed, while the McKenzie method did not affect cortisolaemia significantly. We did not notice any difference in testosterone levels between the two groups. Both treatment methods contributed towards clinical improvement in our patients, represented by the drop in VAS and NDI scores. We also observed a biochemical improvement: decreased cortisol level in the group treated with suboccipital relaxation. Due to the important role of testosterone and cortisol in the pathogenesis of chronic pain, our study should be the pilot experience on their use as markers in CSD.

Key words: cortisol; testosterone; McKenzie therapy; suboccipital relaxation
\end{abstract}

Med Res J 2020; 5 (1): 34-40

\section{Introduction}

In recent years, cervical spine dysfunction (CSD) has become a significant economic and health problem $[1,2]$. The development of utility electronics, sedentary mode of life and locomotion, coupled with a lack of health awareness are the key factors contributing to vertebral dysfunctions [3]. Nowadays, CSD is regarded as a common disease and includes a group of symptoms with pain, limited mobility, and tenderness [4]. Furthermore, the early symptoms are often ignored or masked by the use of easily available over-the-counter (OTC) analgesics [5]. Proper spine functioning is affected by its shape $[1,6]$, vertebral muscle tension, vertebral ligaments, joints, discs, nerves, and sensory organs. Each of these elements plays a substantial role in maintaining somatosensory integrity [7]. Impairment of any of above-mentioned components may contribute towards certain types of spine dysfunction, i.e. overloading of spinal joints causes instability of trunk segments.

Common causes of the early stages of CSD include cervical spine kyphosis and loss of cervical lordosis. These changes of the cervical segment can not only displace the dural sac, but also affect the muscles and the ligaments of the posterior, anterior, and central column. Subsequent posterior displacement of the nucleus pulposus may result in protrusion of the annulus fibrosus towards the spinal canal. This may lead to nerve root remodelling and increases the pressure exerted on the nerve structures [8].

The compression of the nerve roots impairs proper functioning of the brachial and cervical plexuses. This 
leads to symptoms such as pain, paraesthesia, muscle weakness, or weakening of motion range in one or more segments. It may also cause a dysfunction of the peripheral nerves [9].

Re-establishing proper posture, as close as possible to the physiological cervical lordosis, is one of the first steps of CSD treatment [6]. The restoration of proprioception in the suboccipital area is another key factor in the process. Adequate functioning of proprioception receptors relies not only on proper circulation and muscle tone, but also on patient education regarding correct posture. These therapeutic goals can be achieved using two methods: mechanical diagnosis and therapy (MDT, also called the McKenzie method) and suboccipital relaxation [10].

The idea behind the McKenzie method (MDT) is to restore the two basic motion patterns in the cervical spine - retraction and hyperextension. Retraction is the backward head movement which creates lordosis. Hyperextension affects the displaced nucleus pulposus [11] and relaxes the posterior ligament structures, thus relieving the pressure exerted on the nerves [12]. In some patients, therapy also includes cervical spine traction.

Suboccipital relaxation is an osteopathic method targeting the structures of the craniospinal region as well as the surrounding soft tissue [13]. It results in relaxation of these structures, lowers the muscle tone, and reduces blood stasis in the now relaxed suboccipital and perivertebral muscles [14].

The purpose of our study was to asses two methods of physical therapy for CSD: suboccipital relaxation and the McKenzie method. Their effect on the perceived pain level and life quality was evaluated using the VAS and NDI scales. Additionally, we analysed salivary concentrations of chronic pain biomarkers to evaluate the results of the examined methods.

\section{Material and methods}

Study was designed according to the CONSORT statement [15]. Eighty-six adult patients who met the inclusion criteria were divided into two groups: Group A and Group B. Group A included 42 patients (34 women, eight men) treated using the McKenzie method. Group B consisted of 44 patients (33 women, 11 men) subjected to suboccipital relaxation. The average age was 50.1 years $(S D=10.4$, range $26-64)$ in the first group and 48.1 years $(S D=10.8$, range $26-64)$ in the second group. There were no statistically significant differences in age or gender ratio between the two groups $(p>0.05)$.

No changes of trial design were made during the experiment. We assumed that the sample size should include 40 patients, which is the medium number of cervical spondylosis cases in our centre per month.

In each case, CSD was confirmed using magnetic resonance imaging (MRI). Inclusion criteria comprised diagnosis of cervical spine pain lasting more than four weeks, loss or restriction of movement in the cervical spine, spondylosis and spondyloarthrosis with persistent or intermittent pain or CSD-related headaches, patient's age > 18 years, and signed consent for participation. We excluded patients with cervical spine or head trauma, muscle weakness caused by a critical stenosis of the spinal or root canal, coexistence of carpal tunnel syndrome or ulnar nerve compression, constant pain-relieving pharmacotherapy, drug abuse, neoplastic diseases, myasthenia gravis, steroid therapy, antibiotic or antiviral therapy, Cushing's syndrome, Arnold-Chiari syndrome, syringomyelia, and other congenital defects of the head and the cervical area. Lack of informed consent was also an excluding factor. The study was approved by the Committee on Research Ethics of the Silesian Chamber of Physicians (Komisja Bioetyczna Śląskiej Izby Lekarskiej No. 46/2015).

Every patient underwent three treatment sessions, one in every three weeks. The pain assessment was performed two times using the VAS scale - at the beginning of the experiment and after the last session. The disability level was evaluated with the NDI scale, before the first treatment session and after the last one. To assess the hormone levels, saliva was collected using "salivette" type test tubes. The patients had to abstain from certain activities for 20 minutes before the sample collection. The restrictions included eating, cigarette smoking, tooth brushing, gum chewing, and drinking. After collection the samples were centrifuged and frozen at $-85^{\circ} \mathrm{C}$ for storage. The laboratory examinations were performed in the Chair and Department of Medical and Molecular Biology of the Faculty of Medical Sciences in Zabrze, Medical University of Silesia in Katowice.

\section{VAS (visual analogue scale)}

The VAS is a reliable tool for the assessment of pain intensity. The scale is represented by a $10 \mathrm{~cm}$ coloured ruler, with its ends defining the extreme limits. The left margin represents a lack of pain, while the right one represents the strongest pain imaginable. Patients indicate the pain level by using their finger $[16,17]$.

\section{NDI (neck disability index)}

The NDI is a questionnaire to be completed by the patient. It includes 10 questions assessing the impact of neck pain on daily life activities. The index can be used as a self-report measure of neck pain [18]. 


\section{Assessment of testosterone and cortisol levels}

In order to evaluate the testosterone and cortisol levels, commercial ELISA tests (DiaMetria Italy) were used. Absorbance assessment was performed using the $\mu$ Quant reader (BioTek USA), and the obtained data were analysed with KCJunior software (BioTek USA). Method sensitivity for cortisol was $0.12 \mathrm{ng} / \mathrm{mL}$, and for testosterone it was $3.28 \mathrm{pg} / \mathrm{mL}$. Method inaccuracy for cortisol was $6.2 \%$, and for testosterone it was $7.9 \%$.

\section{Statistical analysis}

Statistical analysis of the data was performed using Statistica $13 \mathrm{PL}$ software. The patient age was expressed as the average $+/$ - standard deviation (SD). To compare the age in both groups, Student's t-test was used. The Shapiro-Wilk test was used to determine distributions of variables. Non-normal variables were presented as median with interquartile range. To compare non-parametric variables the Mann Whitney U-test was performed for comparison of groups and the Wilcoxon test to assess differences between initial and final values of analysed variables. Two-way ANOVA for repeatable measurements was performed to assess the effect of gender on testosterone concentration before and after treatment. $P$ values $<0.05$ were considered significant.

\section{Results}

We observed a significant decrease in perceived pain expressed as VAS score in both groups (Tab. 1, Fig. 1). There was no significant difference in VAS values

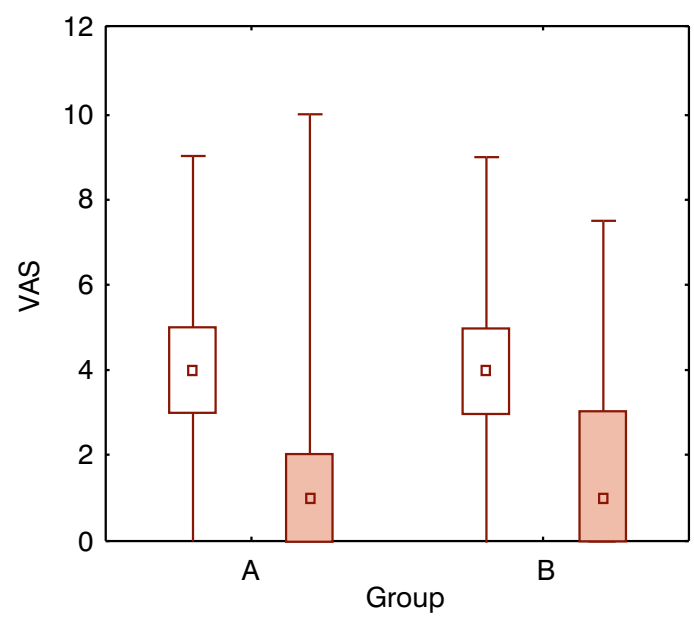

Before treatment

after treatment

Figure 1. VAS scores among examined groups before and after therapy. Data presented as median with interquartile range; VAS - visual analogue scale

between groups at the beginning of the experiment, nor after treatment (Tab. 2). A significant drop in salivary testosterone levels was observed among patients who underwent McKenzie treatment (Tab. 1).

Significant decreases of NDI values were observed in both groups (Tab. 1, Fig. 2). There was no difference in NDI values between groups at the beginning of experiment and at the outcome (Tab. 2).

We found no significant decrease of testosterone level in the group treated with suboccipital relaxation. There was no difference in testosterone concentration

Table 1. VAS, NDI, testosterone, and cortisol values before and after treatment

\begin{tabular}{|c|c|c|c|c|c|}
\hline \multicolumn{6}{|l|}{ McKenzie Method } \\
\hline \multirow[b]{2}{*}{ VAS } & \multicolumn{2}{|c|}{ Before treatment } & \multicolumn{2}{|c|}{ After treatment } & \multirow{2}{*}{$\begin{array}{l}\mathbf{p} \\
<0.001\end{array}$} \\
\hline & 4 & $(3-5)$ & 1 & $(0-2)$ & \\
\hline NDI & 15 & $(10-19)$ & 9 & $(6-13)$ & $<0.001$ \\
\hline Testosterone [pg/mL] & 53.4 & $(49.7-59.7)$ & 55.8 & $(49.8-59.8)$ & $<0.001$ \\
\hline Cortisol [ng/mL] & 22.5 & $(19.5-26.8)$ & 23.5 & $(22.3-26.7)$ & 0.95 \\
\hline \multicolumn{6}{|c|}{ Suboccipital relaxation } \\
\hline & \multicolumn{2}{|c|}{ Before treatment } & \multicolumn{2}{|c|}{ After treatment } & $\mathbf{p}$ \\
\hline VAS & 4 & $(3-5)$ & 1 & $(0-3)$ & $<0.001$ \\
\hline NDI & 15 & $(10-19)$ & 9 & $(6-13)$ & $<0.001$ \\
\hline Testosterone [pg/mL] & 55.3 & $(49.85-59.8)$ & 53.8 & $(51.3-55.4)$ & 0.07 \\
\hline Cortisol [ng/mL] & 22.6 & $(19.5-26.6)$ & 18.15 & $(17.2-19.7)$ & $<0.001$ \\
\hline
\end{tabular}


Table 2. Comparison of values of examined parameters among groups

\begin{tabular}{|c|c|c|c|c|c|}
\hline \multicolumn{6}{|l|}{ Before treatment } \\
\hline \multirow[b]{2}{*}{ VAS } & \multicolumn{2}{|c|}{ McKenzie method } & \multicolumn{2}{|c|}{ Suboccipital relaxation } & \multirow{2}{*}{$\begin{array}{l}\mathbf{p} \\
0.86\end{array}$} \\
\hline & 4 & $(3-5)$ & 4 & $(3-5)$ & \\
\hline NDI & 15 & $(10-19)$ & 15 & $(10-19)$ & 0.65 \\
\hline Testosterone & 53.4 & $(49.7-59.7)$ & 55.3 & $(49.85-59.8)$ & 0.50 \\
\hline Cortisol [ng/mL] & 22.5 & $(19.5-26.8)$ & 22.6 & $(19.5-26.6)$ & 0.97 \\
\hline \multicolumn{6}{|l|}{ After treatment } \\
\hline & \multicolumn{2}{|c|}{ McKenzie method } & \multicolumn{2}{|c|}{ Suboccipital relaxation } & $\mathbf{p}$ \\
\hline VAS & 1 & $(0-2)$ & 1 & $(0-3)$ & 0.70 \\
\hline NDI & 9 & $(6-13)$ & 9 & $(6-13)$ & 0.59 \\
\hline Testosterone [pg/mL] & 55.8 & $(49.8-59.8)$ & 53.8 & $(51.3-55.4)$ & $<0.001$ \\
\hline Cortisol [ng/mL] & 23.5 & (22.3-26.7) & 18.15 & $(17.2-19.7)$ & 0.09 \\
\hline
\end{tabular}

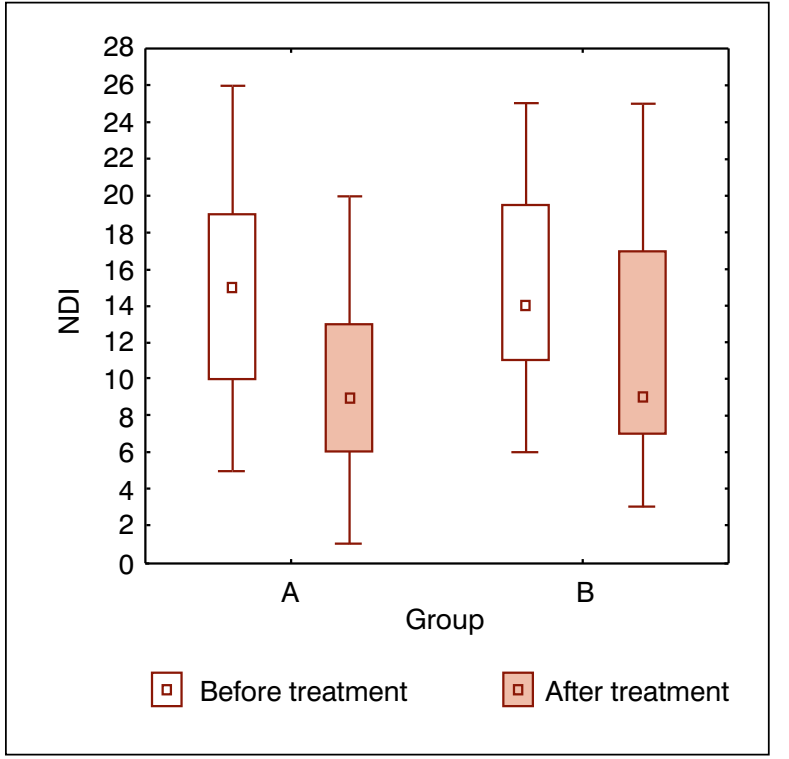

Figure 2. NDI scores among examined groups before and after therapy. Data presented as median with interquartile range; NDI — neck disability index

between the examined groups at first measurement; however, its concentration after therapy was significantly lower in the group that underwent suboccipital relaxation (Tab. 2, Fig. 3).

We did not observe significant changes in cortisol concentration among patients who underwent McKenzie Therapy (Tab. 1, Fig. 4). A significant drop in hormone concentration was observed in the suboccipital relaxation group. There were no significant differences in cortisol concentrations between the examined groups at the beginning and after therapy.

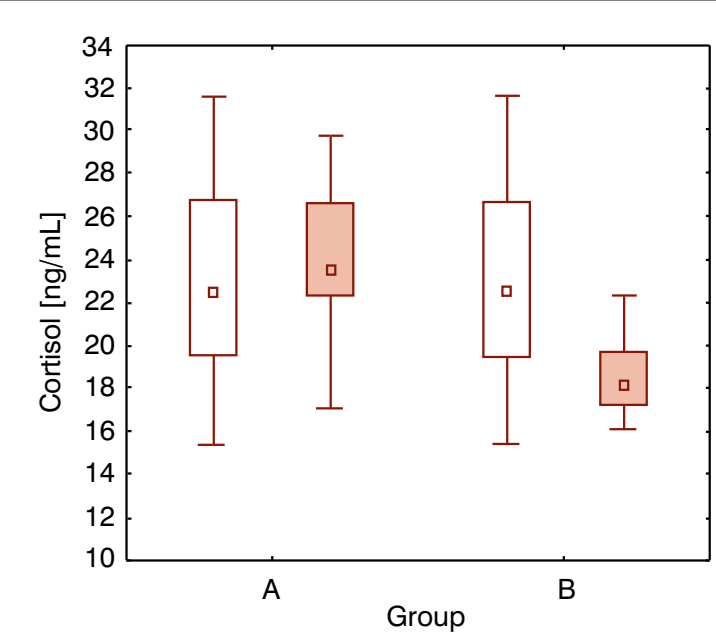

Before treatment

After treatment

Figure 3. Salivary testosterone levels among examined groups before and after therapy. Data presented as median with interquartile range

\section{Discussion}

Both methods compared in our paper are effective and life-quality improving treatment options for patients with CSD. The McKenzie method is an efficient means of achieving relaxation of cervical muscles and diminishing tension headaches [19]. Kjellman et al. [20] reported a significant improvement of quality of life in patients treated using this method. However, Clare et al. [10] in their systematic review did not prove the unambiguous efficiency of the McKenzie method due to a lack 


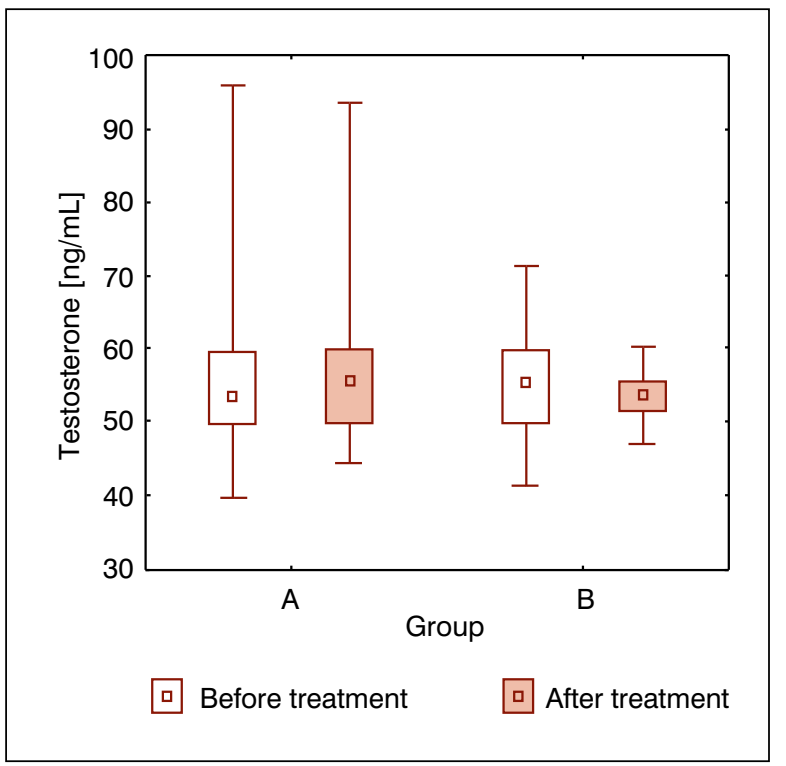

Figure 4. Salivary cortisol levels among examined groups before and after therapy. Data presented as median with interquartile range

of scientific evidence. A decrease in the VAS scores in patients who underwent the McKenzie therapy was reported by Moffet et al. [21]. The authors compared it to physiotherapy and did not report a significant advantage of either method. Suboccipital relaxation therapy has also been found to be effective in CSD, and its efficiency has been observed in the management of tension headaches [22]. The latter was also reported by Jung Yang et al. [23].

In our study, a significant decrease in perceived pain level over time was observed in both groups, without any significant differences in pain relapse. However, a proper verification of these results would require a larger sample size. Both methods were shown to not only alleviate the pain related to the CSD, but also to increase the quality of life evaluated using the NDI score. We did not observe any differences in efficiency between them. Currently, scientific papers on the subject are scarce, out of which only a few attempted scientific validation by evaluating the levels of biochemical pain indicators. In an attempt to objectify our results, we did not limit them to a subjective assessment of pain perceived by the patient, but we also measured the testosterone and cortisol levels.

Chronic pain may not only alter cortisol excretion, exacerbating the symptoms of CSD [24], but may also affect the circadian rhythm of hormones. Analgesia has been observed to normalise serum cortisol levels [25]. In our study, we attempted to verify whether both methods are equally efficient in pain alleviation. We observed a significant cortisol drop only among patients who underwent suboccipital relaxation therapy. No differences in cortisol level were found in group that underwent the McKenzie method treatment. However, longer observation of a study group comprising a larger sample size would be needed to confirm this effect; therefore, we cannot rule out the possibility of the decrease being accidental. Salivary cortisol level increase after cervical manipulations was also reported in a randomised, controlled trial performed by Valera-Calero et al. [26]. Similar results were reported by Plaza-Manzano et al. However, they did not observe differences in pre- and post-treatment cortisol level [27]. Nonetheless, it should be noted that the authors used peripheral blood samples to measure the hormone levels while collecting them, which may be associated with additional stress in the patient. Further research is needed in order to verify the data that were obtained. Analysis of salivary cortisol levels in patients was performed by Moyer et al. in their meta-analysis. The authors concluded that massage has no significant effect on the hormone concentration, based on 18 studies [28]. However, their work analysed studies of patients suffering from various medical conditions. In order to precisely assess the CSD-related cortisol fluctuations, further studies are needed [28]. The authors emphasise that a lack of influence on the cortisol levels does not prove the ineffectiveness of manual therapy. For instance, the high-velocity low-amplitude (HVLA) chiropractic method was shown to have no influence on the hormone concentration [29]. Sobas et al. [30] consider cortisol level to be an unreliable pain biomarker due to its changeability. Many other factors, such as the time of the day or stress, may cause alterations in cortisol levels.

Testosterone and cortisol are two hormones that play an important role in the pathogenesis of chronic pain. The latter is synthesised in the adrenal cortex, and its release follows a characteristic circadian rhythm [31-33]. It is responsible for proper glucose metabolism, regulation of the protein catabolism, and maintenance of functions of the liver and the gastrointestinal tract. Moreover, cortisol exhibits immunosuppressive properties and can be used as an anti-inflammatory drug. It is also an important element of natural response to stress of the human body. A short-term increase of cortisol levels is considered beneficial [34]. However, prolonged stress may disrupt these physiological mechanisms, and chronic pain has been identified as an underlying cause of an abnormal stress response [35]. The early anti-inflammatory effect of cortisol is eventually suppressed due to steroid receptor downregulation, subsequently disrupting synthesis of the hormone. This may result in destabilisation of its circadian rhythm [36], leading to either hyper- or hypocortisolism. The dysfunctions in cortisol excretion ultimately lead to the lowering of the pain threshold, exacerbating the clinical symptoms [24]. 
Testosterone is synthesised in the gonads and the adrenal cortex. Its level correlates negatively with exposure to stress [37]. A suppressing effect of testosterone on the hypothalamic-pituitary-adrenal (HPA) axis has been observed [24]. Furthermore, it has been proven to affect pain perception in patients with fibromyalgia and other diseases [38]. There is a correlation between gonadectomy and increased susceptibility to pain in rats $[39,40]$.

The analgesic effect of testosterone was first described in the 1990s [41]. The hormone exerts a suppressing effect on inflammatory mediators, as observed during antiandrogen therapy [42]. Testosterone also [38] exhibits a central effect - its concentration is positively correlated with middle frontal cortex activity, reducing pain in patients of both sexes [43]. The results of the aforementioned studies indicate that testosterone plays an important role in pain diminishment. In our study we observed significant drop of testosterone concentration among patients who underwent the McKenzie method and no significant changes among patients treated with suboccipital relaxation. We could not find any studies evaluating testosterone levels in cervical spine disorders. It is considered that exposure to stress factors; such as chronic pain is associated with a lower testosterone concentration. Some authors suggest that pain perception is negatively correlated with testosterone levels [44].

\section{Conclusions}

In our study, both methods were found to positively affect the condition of the patients as represented by the improvement in VAS and NDI scores. No significant advantage of either method was observed. While there were no differences in testosterone concentration between the methods, suboccipital relaxation was associated with a significant decrease in cortisol level. However, we were unable to assess which method is more efficient. To the best of our knowledge, to date there have been no other studies comparing the McKenzie method with suboccipital relaxation, nor correlating them with testosterone and cortisol levels. We were unable to indicate superiority of either method due to the limitations of our work: small sample sizes and a relatively short period of observation. Nonetheless, our results indicate a significant improvement in the life quality of the patients, represented by a drop in the VAS and NDI scores. These results may be considered as a background for further research evaluating the use of the suboccipital relaxation and the McKenzie method in cervical spine disorders.

\section{References}

1. Tatu L, Jost WH. Anatomy and cervical dystonia : "Dysfunction follows form". J Neural Transm (Vienna). 2017; 124(2): 237-243, doi: 10.1007/s00702-016-1621-7, indexed in Pubmed: 27624726.

2. Thompson DP, Woby SR. Acceptance in chronic neck pain: associations with disability and fear avoidance beliefs. Int J Rehabil Res. 2017; 40(3): 220-226, doi: 10.1097/MRR.0000000000000230, indexed in Pubmed: 28445326.

3. Cohen SP. Epidemiology, diagnosis, and treatment of neck pain. Mayo Clin Proc. 2015; 90(2): 284-299, doi: 10.1016/i.mayocp.2014.09.008, indexed in Pubmed: 25659245.

4. Weber P, Corrêa EC, Ferreira Fd, et al. Cervical spine dysfunction signs and symptoms in individuals with temporomandibular disorder. J Soc Bras Fonoaudiol. 2012; 24(2): 134-139, doi: 10.1590/s217964912012000200008, indexed in Pubmed: 22832680.

5. Ferrara LA. The biomechanics of cervical spondylosis. Adv Orthop 2012; 2012: 493605, doi: 10.1155/2012/493605, indexed in Pubmed: 22400120 .

6. Lippa L, Lippa L, Cacciola F. Loss of cervical lordosis: What is the prognosis? J Craniovertebr Junction Spine. 2017; 8(1): 9-14, doi: 10.4103/0974-8237.199877, indexed in Pubmed: 28250631.

7. Cramer GD, Darby SA. Basic and clinical anatomy of the spine, spinal cord and ANS, 2nd edition. Elsevier Mosby, St. Louis 2005.

8. Aerbi M, Aebi M, Arlet V. et al. AO ji zhu shou ce, 1st edition. Shan dong ke xue ji shu chu ban she, Jinan 2010

9. Benzel EC. Spine surgery: Techniques, complication avoidance, and management, 2nd edition. Churchill Livingstone, New York 2005.

10. Clare H, Adams R, Maher C. A systematic review of efficacy of McKenzie therapy for spinal pain. Australian Journal of Physiotherapy. 2004; 50(4): 209-216, doi: 10.1016/s0004-9514(14)60110-0.

11. Werneke M, Hart DL, Cook D. A descriptive study of the centralization phenomenon. A prospective analysis. Spine (Phila Pa 1976). 1999; 24(7): 676-683, doi: 10.1097/00007632-199904010-00012, indexed in Pubmed: 10209797.

12. Mercer S, Bogduk N. The ligaments and annulus fibrosus of human adult cervical intervertebral discs. Spine (Phila Pa 1976). 1999; 24(7): 619-26; discussion 627, doi: 10.1097/00007632-19990401000002, indexed in Pubmed: 10209789

13. Franke H, Franke JD, Fryer G. Osteopathic manipulative treatment for nonspecific low back pain: a systematic review and meta-analysis. BMC Musculoskelet Disord. 2014; 15: 286, doi: 10.1186/14712474-15-286, indexed in Pubmed: 25175885.

14. Haller $\mathrm{H}$, Lauche $\mathrm{R}$, Cramer $\mathrm{H}$, et al. Craniosacral Therapy for the Treatment of Chronic Neck Pain: A Randomized Sham-controlled Trial. Clin J Pain. 2016; 32(5): 441-449, doi: 10.1097/AJP.0000000000000290, indexed in Pubmed: 26340656.

15. Schulz KF, Altman DG, Moher D, et al. CONSORT Group. CONSORT 2010 Statement: updated guidelines for reporting parallel group randomised trials. BMC Med. 2010; 8: 18, doi: 10.1186/1741-7015-8-18, indexed in Pubmed: 20334633

16. Faiz K. VAS - visuell analog skala. Tidsskrift for Den norske legeforening. 2014; 134(3): 323-323, doi: 10.4045/tidsskr.13.1145.

17. Heller GZ, Manuguerra M, Chow R. How to analyze the Visual Analogue Scale: Myths, truths and clinical relevance. Scand J Pain. 2016; 13: 67-75, doi: 10.1016/j.sjpain.2016.06.012, indexed in Pubmed: 28850536 .

18. MacDermid JC, Walton DM, Avery S, et al. Measurement properties of the neck disability index: a systematic review. J Orthop Sports Phys Ther. 2009; 39(5): 400-417, doi: 10.2519/jospt.2009.2930, indexed in Pubmed: 19521015.

19. Choi SY, Choi JH. The effects of cervical traction, cranial rhythmic impulse, and Mckenzie exercise on headache and cervical muscle stiffness in episodic tension-type headache patients. J Phys Ther Sci. 2016; 28(3): 837-843, doi: 10.1589/jpts.28.837, indexed in Pubmed: 27134368.

20. Kjellman G, Öberg B. A randomized clinical trial comparing general exercise, McKenzie treatment and a control group in patients with neck pain. J Rehabil Med. 2002; 34(4): 183-190, doi 10.1080/16501970213233.

21. Moffett JK, Jackson DA, Gardiner ED, et al. Randomized trial of two physiotherapy interventions for primary care neck and back pain patients: 'McKenzie' vs brief physiotherapy pain management. Rheumatology (Oxford). 2006; 45(12): 1514-1521, doi: 10.1093/rheuma$\underline{\text { tology/kel339, indexed in Pubmed: } 17062645}$ 
22. Espí-López G, Arnal-Gómez A, Arbós-Berenguer T, et al. Effectiveness of Physical Therapy in Patients with Tension-type Headache Literature Review. J Jpn Phys Ther Assoc. 2014; 17(1): 31-38, do 10.1298/iipta.vol17 005.

23. Yang DJ, Kang DaH. Comparison of muscular fatigue and tone of neck according to craniocervical flexion exercise and suboccipital relaxation in cervicogenic headache patients. J Phys Ther Sci. 2017; 29(5) 869-873, doi: 10.1589/ipts.29.869, indexed in Pubmed: 28603362.

24. Fries $E$, Hesse J, Hellhammer J, et al. A new view on hypocortisolism. Psychoneuroendocrinology. 2005; 30(10): 1010-1016, doi: 10.1016/ psyneuen 2005.04.006, indexed in Pubmed: 15950390.

25. Tennant F, Hermann L. Normalization of serum cortisol concentration with opioid treatment of severe chronic pain. Pain Med. 2002; 3(2): 132-134, doi: 10.1046/j.1526-4637.2002.02019.x, indexed in Pubmed 15102160 .

26. Valera-Calero A, Lluch Girbés E, Gallego-Izquierdo T, et al. Endocrine response after cervical manipulation and mobilization in people with chronic mechanical neck pain: a randomized controlled trial. Eur J Phys Rehabil Med. 2019; 55(6): 792-805, doi: 10.23736/S19739087.19.05475-3, indexed in Pubmed: 30621368.

27. Plaza-Manzano G, Molina-Ortega F, Lomas-Vega R, et al. Changes in biochemical markers of pain perception and stress response afte spinal manipulation. J Orthop Sports Phys Ther. 2014; 44(4): 231-239, doi: 10.2519/jospt.2014.4996, indexed in Pubmed: 24450367.

28. Moyer CA, Seefeldt L, Mann ES, et al. Does massage therapy reduce cortisol? A comprehensive quantitative review. J Bodyw Mov Ther 2011; 15(1): 3-14, doi: 10.1016/i.jbmt.2010.06.001, indexed in Pubmed: 21147413

29. Whelan T, Dishman J, Burke J, et al. The effect of chiropractic manipulation on salivary cortisol levels. J Manipulative Physiol Ther. 2002 25(3): 149-153, doi: 10.1067/mmt.2002.122328.

30. Sobas EM, Reinoso R, Cuadrado-Asensio R, et al. Reliability o Potential Pain Biomarkers in the Saliva of Healthy Subjects: Inter-Individual Differences and Intersession Variability. PLoS One. 2016 11(12): e0166976, doi: 10.1371/journal.pone.0166976, indexed in Pubmed: 27907037.

31. Chan S, Debono M. Replication of cortisol circadian rhythm: new advances in hydrocortisone replacement therapy. Ther Adv Endocrinol Metab. 2010; 1(3): 129-138, doi: 10.1177/2042018810380214, indexed in Pubmed: 23148157.

32. Patacchioli FR, Monnazzi P, Simeoni S, et al. Salivary cortisol, dehydroepiandrosterone-sulphate (DHEA-S) and testosterone in women with chronic migraine. J Headache Pain. 2006; 7(2): 90-94, doi: 10.1007/s10194-006-0274-6, indexed in Pubmed: 16575505.

33. Krieger DT, Allen W, Rizzo F, et al. Characterization of the normal temporal pattern of plasma corticosteroid levels. J Clin Endocrinol Metab. 1971; 32(2): 266-284, doi: 10.1210/jcem-32-2-266, indexed in Pubmed: 4321505.

34. Pocock G, Richards Cd, Richards D. Human physiology. Oxford Univ Press, Oxford 2017

35. Hannibal KE, Bishop MD. Chronic stress, cortisol dysfunction, and pain: a psychoneuroendocrine rationale for stress management in pain rehabilitation. Phys Ther. 2014; 94(12): 1816-1825, doi: 10.2522/pti.20130597, indexed in Pubmed: 25035267.

36. Godfrey KM, Strachan E, Dansie E, et al. Salivary cortisol and cold pain sensitivity in female twins. Ann Behav Med. 2014; 47(2): 180-188, doi: 10.1007/s12160-013-9532-4, indexed in Pubmed: 23955075.

37. Van den Berghe $\mathrm{G}$. The neuroendocrine response to stress is a dynamic process. Best Pract Res Clin Endocrinol Metab. 2001; 15(4): 405419, doi: 10.1053/beem.2001.0160, indexed in Pubmed: 11800514.

38. White HD, Robinson TD. A novel use for testosterone to treat central sensitization of chronic pain in fibromyalgia patients. Int Immunopharmacol. 2015; 27(2): 244-248, doi: 10.1016/i.intimp.2015.05.020, indexed in Pubmed: 26004314

39. Forman LJ, Tingle V, Estilow $\mathrm{S}$, et al. The response to analgesia testing is affected by gonadal steroids in the rat. Life Sciences. 1989; 45(5): 447-454, doi: 10.1016/0024-3205(89)90631-0.

40. Aloisi AM, Ceccarelli I, Fiorenzani P, et al. Testosterone affects formalin-induced responses differently in male and female rats. Neurosci Lett. 2004; 361(1-3): 262-264, doi: 10.1016/i.neulet.2003.12.023, indexed in Pubmed: 15135943

41. Aloisi A, Ceccarelli I, Lupo C. Behavioural and hormonal effects of restraint stress and formalin test in male and female rats. Brain Research Bulletin. 1998; 47(1): 57-62, doi: 10.1016/s0361-9230(98)00063-x.

42. Vodo S, Bechi N, Petroni A, et al. Testosterone-induced effects on lipids and inflammation. Mediators Inflamm. 2013; 2013: 183041, doi: 10.1155/2013/183041, indexed in Pubmed: 23606790.

43. Choi J, Park S, Kim YH, et al. Different Brain Activation Patterns to Pain and Pain-related Unpleasantness during the Menstrual Cycle. Anesthesiology. 2006; 105(1): 120-127, doi: 10.1097/00000542200607000-00021.

44. Choi JC, Chung MI, Lee YD, Modulation of pain sensation by stress-related testosterone and cortisol. Anaesthesia. 2012; 67(10): 1146-1151, doi: 10.1111/j.1365-2044.2012.07267.x, indexed in Pubmed: 22804789. 